\title{
Global Swing Instability in the New England Power Grid Model
}

$\operatorname{AUTHOR}(S):$

Susuki, Yoshihiko; Mezi, Igor; Hikihara, Takashi

\section{CITATION:}

Susuki, Yoshihiko ... [et al]. Global Swing Instability in the New England Power Grid Model. Proceedings of the 2009 American Control Conference (ACC2009) 2009: 3446-3451

\section{ISSUE DATE:}

2009-06

URL:

http://hdl.handle.net/2433/89657

\section{RIGHT:}

(c) 2009 AACC. Personal use of this material is permitted. However, permission to reprint/republish this material for advertising or promotional purposes or for creating new collective works for resale or redistribution to servers or lists, or to reuse any copyrighted component of this work in other works must be obtained from the IEEE. 


\title{
Global Swing Instability in the New England Power Grid Model
}

\author{
Yoshihiko Susuki, Igor Mezić, Takashi Hikihara
}

\begin{abstract}
Global swing instability is an undesirable and emergent phenomenon of synchronous machines in a power grid, implying that most of the machines in the system simultaneously lose synchronism with the rest of the grid after being subjected to a finite and local disturbance. Recently we reported that global instability occurred in the classical model of swing dynamics in the New England power grid model. This paper analyzes the global instability in the New England power grid model. We show that the proper orthonormal decomposition and the Galerkin method for model reduction can determine a dynamical mechanism responsible for the global instability. These methods applied in this paper make it possible to find the occurrence of global instability in real power grids.
\end{abstract}

Index Terms-power systems, stability, POD, Galerkin method

\section{INTRODUCTION}

We studied in [1] global instability of short-term (0 to 10 seconds [2]) swing dynamics in multi-machine power grids. Global instability is an undesirable and emergent phenomenon of synchronous machines in a power grid, implying that a group of machines in the grid simultaneously loses synchronism with the rest of the grid after being subjected to a finite, possibly local disturbance. Global instability is related to three known phenomena in transient stability: local plant mode oscillation, inter-area mode instability, and multi-swing instability in short-term regime [2]. One goal of our study is to find a dynamical mechanism that causes widespread blackouts of real power grids, e.g., 2003 blackouts in North America and Europe [3]. A complex power grid can be decomposed into a large set of strongly innerconnected units or minimal grids that are joined via a weak interconnection. Such decomposition is possible with the methods developed in [4], [5]. An unstable behavior of one unit grid affects the other unit grids via the interconnection and, in the worst case, de-stabilizes some of them. This can repeatedly occur and cause the propagation of instabilities in the entire grid. This physical view provides a hint to answer the question of how a sequence of instabilities, that is, a cascade of instabilities is dynamically organized. In the

This research is supported by JSPS Postdoctoral Fellowships for Research Abroad, 2008-2010.

Y. Susuki is with the Department of Mechanical Engineering at the University of California, Santa Barbara, CA 93106-5070, United States, and is also with the Department of Electrical Engineering at Kyoto University, Katsura, Nishikyo, Kyoto 615-8510 Japan. Email: su suki @ ieee . org

I. Mezic is with the Department of Mechanical Engineering at the University of California, Santa Barbara, CA 93106-5070, United States. Email: mezicdengineering.ucsb.edu

T. Hikihara is with the Department of Electrical Engineering, Photonics and Electronics Science and Engineering Center at $\mathrm{Ky}$ oto University, Katsura, Nishikyo, Kyoto 615-8510 Japan. Email: hikiharaldove.kuee.kyoto-u.ac.jp preceding paper [1], for a simple power grid with strong loop transmission network, we showed that global instability was the escape phenomenon [6] in a dynamical system for the amplitude of nonlinear mode governing collective motion of the machines.

This paper analyzes global instability in the more realistic New England power grid model, which is introduced in [7], [8]. We reported in [1] that global instability occurred in the classical model of swing dynamics for the grid model. The purpose of this paper is to analyze the global instability using numerical simulation, thereby to provide a tool for investigating the occurrence of global instability using simulation outputs of swing dynamics. The above mechanism in [1] is found for a solvable model of global instability and is not applicable to practical models including the so-called classical model [2]. We show that the proper orthonormal decomposition (POD) and the Galerkin method for model reduction [9] can determine a dynamical mechanism responsible for the global instability in the New England grid model. These methods make it possible to find the occurrence of global instability in real power grids.

The rest of this paper is organized as follows. Sec. II introduces global instability in the New England grid model. The simulations presented here are obtained using the classical model in Sec. II-A and a detailed model in Sec. II-B. Sec. III uses the POD for the simulation outputs in Sec. II-A and identifies dominant empirical modes of the phenomenon. Sec. IV applies the Galerkin method to reduction of the classical model used in Sec. II-A and determines a dynamical mechanism responsible for the occurrence of global instability. Sec. V concludes this paper with a summary and discussion.

\section{GlobAL INSTABility}

This section shows global instability of swing dynamics in the New England grid model. The grid model is shown in Fig. 1 and consists of 10 generation units (equivalent 10 synchronous generators), 39 buses, and ac transmission lines. Most of the buses have constant active and reactive power loads. Note that the content in Sec. II-A is reported in the preceding paper [1].

\section{A. Numerical simulation of the classical model}

We assume that bus 39 is the infinite bus in Fig. 1. The short-term swing dynamics of generators 2-10 are represented by the classical model with constant voltage behind 


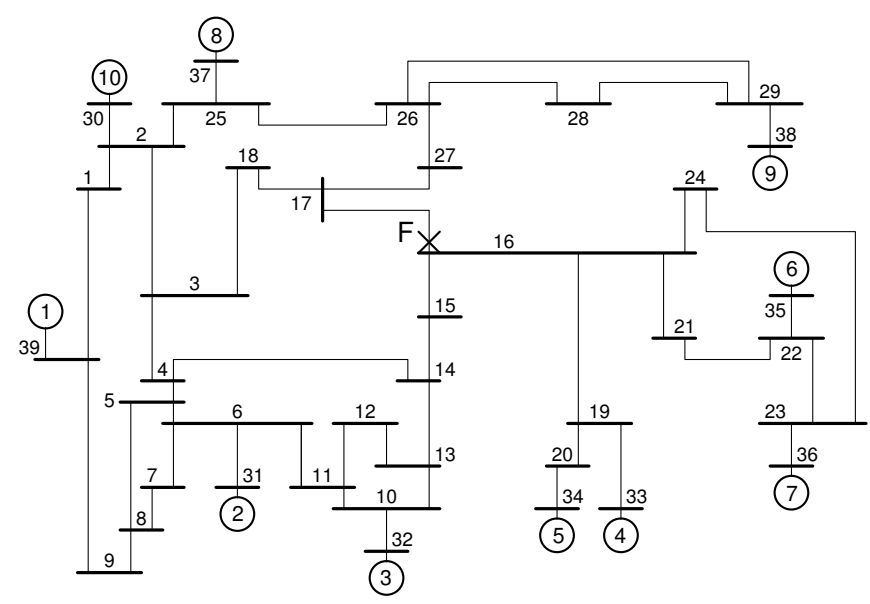

Fig. 1. The New England power grid model [7], [8]

reactance [2] as follows:

$$
\left.\begin{array}{rl}
\frac{\mathrm{d} \delta_{i}}{\mathrm{~d} t}= & \omega_{i}, \\
\frac{H_{i}}{\pi f_{\mathrm{s}}} \frac{\mathrm{d} \omega_{i}}{\mathrm{~d} t}=- & D_{i} \omega_{i}+P_{\mathrm{m} i}-G_{i i} E_{i}^{2}-\sum_{j=1, j \neq i}^{10} E_{i} E_{j} . \\
& \cdot\left\{G_{i j} \cos \left(\delta_{i}-\delta_{j}\right)+B_{i j} \sin \left(\delta_{i}-\delta_{j}\right)\right\},
\end{array}\right\}
$$

where $i=2, \ldots, 10$. The variable $\delta_{i}$ is the rotor angle position of generator $i$ with respect to synchronously reference axis and is in radian; $\omega_{i}$ is the rotor speed deviation of generator $i$ relative to system angular frequency $2 \pi f_{\mathrm{s}}=$ $2 \pi \times(60 \mathrm{~Hz})$ and is in radian per second. The variable $\delta_{1}$ is constant because bus 1 is assumed to be the infinite bus. The parameters $H_{i}, f_{\mathrm{s}}, D_{i}, P_{\mathrm{m} i}, G_{i i}, E_{i}, G_{i j}$, and $B_{i j}$ are in per unit system except for $H_{i}$ and $D_{i}$ in second, and for $f_{\mathrm{s}}$ in Hertz. We assume for short-term rotor angle stability [2] that the mechanical input power $P_{\mathrm{m} i}$ to generator $i$ and the internal voltage $E_{i}$ of generator $i$ are constant. The parameter $H_{i}$ is the inertia constant of generator $i$, and $D_{i}$ is its damping coefficient. $G_{i i}$ is the internal conductance, and $G_{i j}+\mathrm{j} B_{i j}$ the transfer impedance between generators $i$ and $j$. They are the parameters that change with network topology changes. We also model electrical loads in the grid model as lumped passive impedances [2].

We numerically simulate coupled swing dynamics of generators $2-10$. The voltage $E_{i}$ and the initial condition $\left(\delta_{i}(0), \omega_{i}(0)=0\right)$ for generator $i$ are fixed through power flow calculation. The inertia constant $H_{i}$ is fixed at the original value in [8]. We here use the following load condition: the input power $P_{\mathrm{m} i}$ and constant power loads are $50 \%$ at their rating, which setting is used in [10]. The damping $D_{i}$ is fixed at $0.005 \mathrm{~s}$ for each generator. ${ }^{1}$ The constants $G_{i i}$, $G_{i j}$, and $B_{i j}$ are fixed with the original line data in [8] and the power flow calculation. For this simulation we use the following fault condition: each generator operates at a steady

\footnotetext{
${ }^{1}$ When rotor angle deviation $\omega_{i}$ is in per unit system with base $2 \pi f_{\mathrm{s}}$, the damping $D_{i}=0.005 \mathrm{~s}$ is equal to 1.88 in per unit system with its base $1 /\left(2 \pi f_{\mathrm{s}}\right)$
}
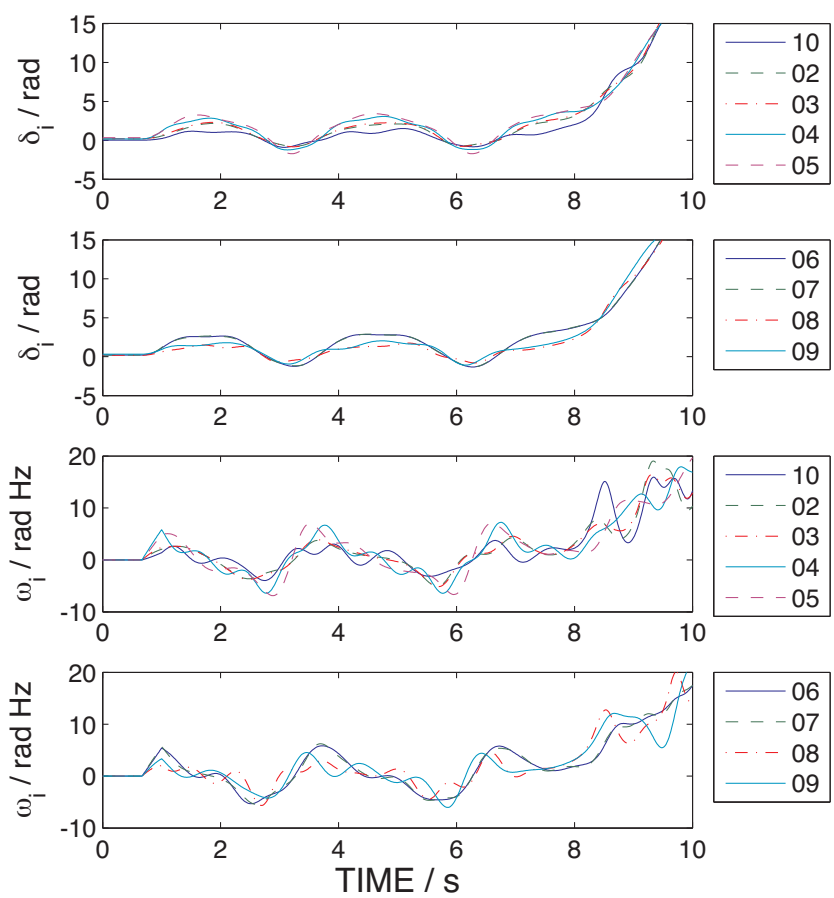

Fig. 2. Global instability in the classical model (1) of swing dynamics in the New England power grid model

condition at $t=0 \mathrm{~s}$, a three-phase fault occurs at point $\mathrm{F}$ near bus 16 at $t=1 \mathrm{~s}-20 /(60 \mathrm{~Hz})=2 / 3 \mathrm{~s}$, and line $16-17$ trips at $t=1 \mathrm{~s}$. The fault duration is 20 cycles of a $60-\mathrm{Hz}$ sine wave. The fault is simulated by adding a small impedance $\left(10^{-7} \mathrm{j}\right)$ between bus 16 and the ground.

Figure 2 shows time responses of rotor angle position $\delta_{i}$ and rotor speed deviation $\omega_{i}$ for the classical model (1) which are reported in [1]. Before $t=2 / 3 \mathrm{~s}$, namely, the onset time of fault, each generator operates at a steady condition. In the fault duration from $t=2 / 3 \mathrm{~s}$ to $1 \mathrm{~s}$, all the generators $2-10$ accelerate apart from their steady conditions. After the line trip at $t=1 \mathrm{~s}$, they respond in an oscillatory manner. These oscillations are bounded during the period from $t=1 \mathrm{~s}$ to $8 \mathrm{~s}$ and then begin to increase simultaneously. That is, each generator loses synchronism with the infinite bus at the same time. The simultaneous increase is a typical feature of global instability.

\section{B. Numerical simulation of a detailed model}

This subsection presents a numerical simulation of global instability based on a detailed model of synchronous generators and controllers. The classical model (1) is normally used for the first swing criterion [2], because second and multi swings may be affected by flux decays and control effects in generators. Such effects cannot be represented by the simple model (1) with constant input power and voltage. Then it is questionable whether the global instability in Fig. 2 persists under their additional effects. Numerical simulation, however, shows that an instability similar to that in Fig. 2 is observed for a detailed model. 
For the development of a detailed model, we assume that the dynamics of internal flux, line transients, and PSS are negligible. This is valid in short-term rotor angle stability [2]. The voltage-current characteristics in transmission lines are represented by the well-known phasor description based on the admittance matrix $Y$. First, we model the dynamics of generator $i$ using the following equations:

$$
\left.\begin{array}{rl}
T_{d 0 i}^{\prime} \frac{\mathrm{d} e_{q i}^{\prime}}{\mathrm{d} t} & =-e_{q i}^{\prime}+e_{f d i}-\left(L_{d i}-L_{d i}^{\prime}\right) i_{d i}, \\
\frac{\mathrm{d} \theta_{i}}{\mathrm{~d} t} & =2 \pi f_{\mathrm{s}} \omega_{i}, \\
\frac{H_{i}}{\pi f_{\mathrm{s}}} \frac{\mathrm{d} \omega_{i}}{\mathrm{~d} t} & =\frac{p_{\mathrm{m} i}}{\omega_{i}}-\left(\phi_{d i} i_{q i}-\phi_{q i} i_{d i}\right),
\end{array}\right\}
$$

where $e_{q i}^{\prime}$ is the voltage behind transient reactance of generator $i$ in per unit system, $\theta_{i}$ is the rotor angle of generator $i$ in radian, and $\omega_{i}$ is its rotor angular speed in per unit system with base $2 \pi f_{\mathrm{s}}$. The parameters $T_{d 0 i}^{\prime}, L_{d i}$, and $L_{d i}^{\prime}$ are constant, and their values are adopted from [8]. The constant $p_{\mathrm{m} i}$ is the mechanical input power to generator $i$ and is regulated by governors. The currents $\left(i_{d i}, i_{q i}\right)$ are given with the terminal voltages $\left(e_{d i}, e_{q i}\right)$ and the admittance matrix $Y$. The magnetic fluxes $\left(\phi_{d i}, \phi_{q i}\right)$ are functions of the currents $\left(i_{d i}, i_{q i}\right)$ and the voltage $e_{q i}^{\prime}: \phi_{d i}=-L_{d i}^{\prime} i_{d i}+e_{q i}^{\prime}$ and $\phi_{q i}=-L_{q i} i_{q i}$. The damping $D_{i}$ is not considered in the detailed model. Now it is supposed that the damping effect is added by the flux decay, AVR and governor actions. Second, we model the effects of excitation controller using the IEEE Type AC4A Excitation System Model [11]. The effect of output limiter is assumed to be invalid, because it does not qualitatively change the following numerical result. The parameters of AVR are also adopted from [11]. The variable $e_{f d i}$ is the output of excitation system. Lastly, we describe the governor action simply by

$$
T_{\mathrm{G}} \frac{\mathrm{d} p_{\mathrm{m} i}}{\mathrm{~d} t}=-K_{\mathrm{G}}\left(\omega_{i}-1\right)-\left(p_{\mathrm{m} i}-P_{\mathrm{m} i}\right),
$$

where $K_{\mathrm{G}}=20$ and $T_{\mathrm{G}}=3 \mathrm{~s} . P_{\mathrm{m} i}$ is the set-point value of mechanical input power to generator $i$. The AVRs and governors for every generator are identical.

We numerically simulate coupled dynamics of generators $2-10$. The bus voltages are fixed through power flow calculation. The inertia constant $H_{i}$ is fixed at the same value as that in Sec. II. The constant $H_{1}$ for generator 1 is fixed at $50000 \mathrm{~s}$. We here use the following load condition: the mechanical input power $P_{\mathrm{m} i}$ and constant power loads are $60 \%$ at their ratings. The elements $G_{i i}, G_{i j}$, and $B_{i j}$ of the matrix $Y$ are obtained with the original line data in [8] and the power flow calculation. For this simulation we use the following fault condition: each generator operates at a steady condition at $t=100 \mathrm{~s}$, a three-phase fault occurs at point $\mathrm{F}$ near bus 16 at $t=102 \mathrm{~s}$, and line 16-17 trips at $t=102 \mathrm{~s}+20 /(60 \mathrm{~Hz}) \approx 102.33 \mathrm{~s}$. The fault duration is 20 cycles of a $60-\mathrm{Hz}$ sine wave. The fault is also simulated by adding a small impedance $\left(10^{-7} \mathrm{j}\right)$ between bus 16 and the ground.
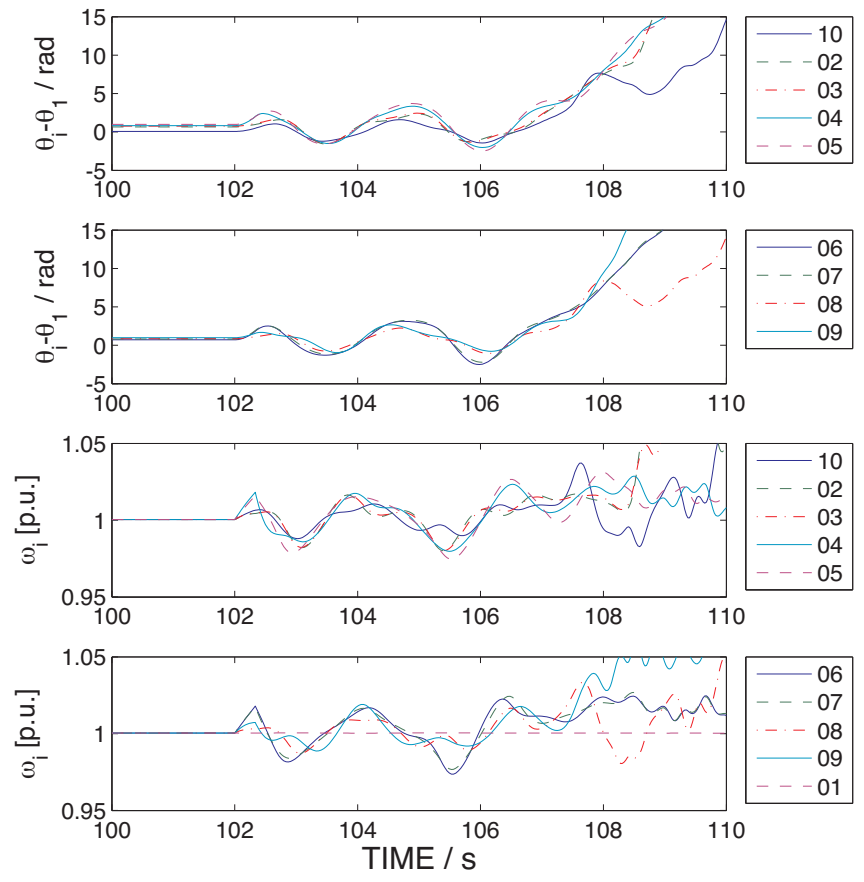

Fig. 3. Global instability in a detailed model of dynamics in the New England power grid model

Figure 3 shows time responses of rotor angle difference $\theta_{i}-\theta_{1}$ and rotor angular speed $\omega_{i}$. At $t=100 \mathrm{~s}$, each generator operates at a steady condition. After the line trip at $t \approx 102.33 \mathrm{~s}$, they respond in an oscillatory manner. These oscillations are bounded for a while and begin to increase simultaneously at $t=107 \mathrm{~s}$. That is, each generator loses synchronism with generator 1 at the same time. The duration between the onsets of fault and instability is about $5 \mathrm{~s}$ and is in short-term regime. This phenomenon is global instability that we have addressed in [1] and this paper. The behaviors of $\omega_{i}$ are different from those in the classical model shown in Fig. 2, because the detailed model includes the effects of flux decay and controllers. The numerical result implies that global instability can be investigated with either the classical model or the detailed continuous model. This paper uses the classical model (1) in what follows.

\section{IDENTIFYING DOMINANT MODES USING THE POD}

The proper orthonormal decomposition (POD) provides a basis for the modal decomposition of an ensemble of functions, such as data obtained in the course of experiments, and provides the most efficient way of capturing the dominant components, if data includes velocity component, with the most energy [9]. This section performs the POD which identifies an energetically dominant set of empirical eigenmodes, called the POD modes, using the simulation outputs of global instability in Fig. 2.

Now we give the definition and procedure of POD. Consider a finite set of simulation outputs of rotor angle positions, $\left\{\delta_{i}\left(n T_{\mathrm{s}}\right)\right\}\left(i=2, \ldots, 10, n=0, \ldots, N_{\mathrm{s}}-1\right)$, 

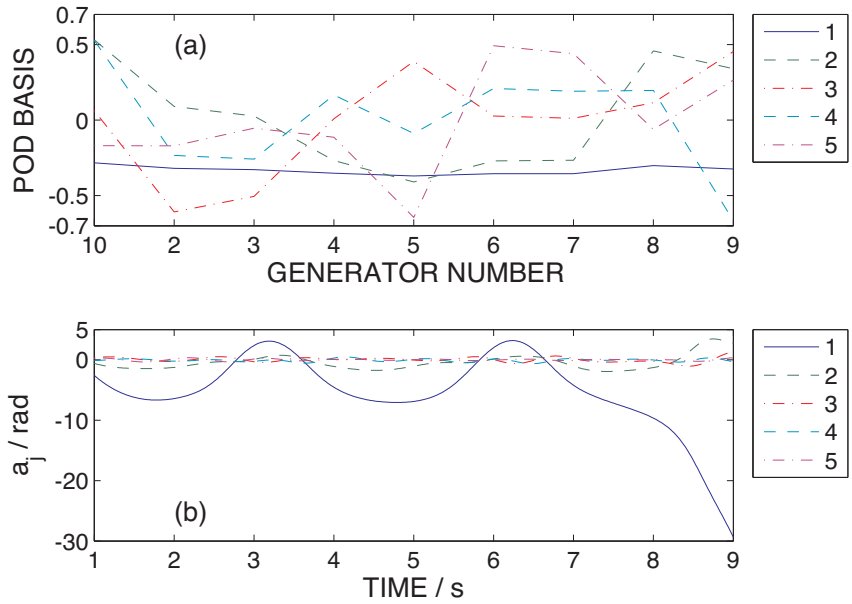

Fig. 4. 1st to 5th POD modes obtained from the simulation outputs of rotor angle positions in global instability of Fig. 2: (a) orthogonal bases $\left\{e_{i j}\right\}$ and (b) coefficients $a_{j}$.

obtained with the classical model (1). $T_{\mathrm{s}}$ is the sampling period of outputs, and $N_{\mathrm{s}}$ is the number of samples. The POD from the outputs is then represented by

$$
\delta_{i}\left(n T_{\mathrm{s}}\right)=\sum_{j=1}^{9} e_{i j} a_{j}\left(n T_{\mathrm{s}}\right) .
$$

The time-invariant bases $\left\{e_{i j}\right\}$ are orthogonal, i.e. $\sum_{j=1}^{9} e_{k j} e_{l j}=\sum_{i=2}^{10} e_{i k} e_{i l}=\Delta_{k l}$, where $\Delta_{k l}$ is the Kronecker delta. The time-varying coefficient $a_{j}$ $(j=1, \ldots, 9)$ in the POD holds the following correlation property: $\left\langle a_{j} a_{k}\right\rangle=\Delta_{j k}\left\langle a_{j}^{2}\right\rangle$, where $\langle\bullet\rangle$ denotes a time average of the data $\{\bullet\}$. The POD modes are also ordered by $\left\langle a_{j}^{2}\right\rangle \geq\left\langle a_{j+1}^{2}\right\rangle$. The POD is numerically obtained by computing correlation matrix $R$ from $\left\{\delta_{i}\left(n T_{\mathrm{s}}\right)\right\}$ and orthonormal bases of $R$.

POD modes are obtained using $N_{\mathrm{s}}=481$ snapshots in the simulation outputs of global instability in Fig. 2. The time interval is $[1 \mathrm{~s}, 9 \mathrm{~s}]$, and $T_{\mathrm{s}}$ is equal to $1 /(60 \mathrm{~Hz})$. Fig. $4(\mathrm{a})$ shows the orthogonal bases of 1st to 5th POD modes. The solid line is for the first POD mode and is almost constant or flat for generator number $i \in\{2, \ldots, 10\}$. The other broken lines are for higher POD modes and are not flat for generator number. Fig. 4(b) also shows the time responses of coefficients $a_{j}$ for these POD modes. The coefficient $a_{1}(t)$ begins to diverge at time $8 \mathrm{~s}$. The onset of divergence corresponds to that of simultaneous increase of $\delta_{i}$ in Fig. 2 . The other $a_{j}(t)$ in Fig. 4(b) do not show any change at the onset time. The results of POD clearly indicate that the first POD mode describes the feature of global instability in Fig. 2.

\section{EXPLORING A DYNAMICAL MECHANISM USING THE GALERKIN METHOD}

The POD showed that the first POD mode with the almost flat base is closely related to the global instability in Fig. 2 . In this section, we indeed show that the occurrence of global instability can be explained through a potential structure in the reduced dynamical system on the sub-space spanned by the first POD base. The content in Sec. IV provides a method of how to find such a potential structure for global instability, in general, for collective dynamics of a group of generators, from the classical model (1) and simulation outputs obtained with it.

Now we review the so-called Galerkin method for reducing high-dimensional nonlinear dynamical systems. This review is based on [12]. Consider the following dynamic model for power grid analysis:

$$
\frac{\mathrm{d} x}{\mathrm{~d} t}=f(x),
$$

where $x$ is the state vector in state space $\mathbb{R}^{K}$, and $f$ is the piecewise continuous vector-valued function defined on $\mathbb{R}^{K}$. We assume that a collection of data of state vector, $\left\{x\left(n T_{\mathrm{S}}\right)\right\}\left(n=0, \ldots, N_{\mathrm{s}}-1\right)$, is obtained with simulation of the model (5). The POD for $\left\{x\left(n T_{\mathrm{s}}\right)\right\}$ is also preliminarily obtained with the correlation matrix $R$ and its orthonormal bases. The POD provides the most efficient way of capturing the dominant modes. Now, let us consider the model (5) on the subspace spanned by the first $k(<K)$ POD modes. We define the projection matrix $P$ of size $K \times k$ by standing the $k$ orthonormal bases of the first $k$ POD modes in lines. The $k$-dimensional state vector $y \in \mathbb{R}^{k}$ is also defined as $y=P^{*} x$, where $P^{*}$ is the real matrix of size $k \times K$ and satisfies $P^{*} P=I$ ( $I$ is the unity matrix of size $\left.k \times k\right)$. Then we have the reduced model as follows:

$$
\frac{\mathrm{d} y}{\mathrm{~d} t}=P^{*} f(P y) .
$$

The state $y$ represents the dynamics of the first $k$ POD modes. By construction, the reduced-order model is expected to approximate the dynamics of the original model (5). The above procedure is called the Galerkin method.

We construct a second-order autonomous dynamical system by applying the Galerkin method to the classical model (1). Let us re-define continuous-time coefficients $a_{j}(t)$ and $b_{j}(t)$ for $j=1, \ldots, 9$ as

$$
\left.\begin{array}{l}
\delta_{i}(t)=\sum_{j=1}^{9} e_{i j} a_{j}(t), \\
b_{j}(t)=\dot{a}_{j}(t), \quad \omega_{i}(t)=\sum_{j=1}^{9} e_{i j} b_{j}(t) .
\end{array}\right\}
$$

where $i=2, \ldots, 10$. The result on POD in Fig. 4 clearly shows that the first POD mode describes the feature of global instability in the original classical model (1). Then, the Galerkin method based on the first POD mode (namely, in the case of $k=1$ ) induces the following second-order 
differential equations:

$$
\begin{aligned}
\frac{\mathrm{d} a_{1}}{\mathrm{~d} t}= & b_{1}, \\
\frac{\mathrm{d} b_{1}}{\mathrm{~d} t}= & \sum_{i=2}^{10} \frac{e_{i 1}}{M_{i}}\left[-D_{i} b_{1} e_{i 1}+P_{\mathrm{m} i}-\sum_{j=1, j \neq i}^{10} E_{i} E_{j} B_{i j} .\right. \\
& \cdot \sin \left(a_{1} e_{i 1}-a_{1} e_{j 1}\right)-\left\{G_{i i} E_{i}^{2}+\sum_{j=1, j \neq i}^{10} E_{i} E_{j} .\right. \\
& \left.\left.\cdot G_{i j} \cos \left(a_{1} e_{i 1}-a_{1} e_{j 1}\right)\right\}\right],
\end{aligned}
$$

where $M_{i}=H_{i} /\left(\pi f_{\mathrm{s}}\right)$ and $e_{11}=0$. Here it should be recalled that the base of the first POD mode is almost flat in Fig. 4(a). If the base is exactly flat, i.e., $e_{i 1}=e_{1}$ for $i=2, \ldots, 10$, then we can re-write the above second-order system equations as

$$
\left.\begin{array}{rl}
\frac{\mathrm{d} a_{1}}{\mathrm{~d} t}= & b_{1}, \\
\frac{\mathrm{d} b_{1}}{\mathrm{~d} t}= & -D\left(e_{1}\right) b_{1}+P\left(e_{1}\right) \\
& -G\left(e_{1}\right) \cos \left(a_{1} e_{1}\right)-B\left(e_{1}\right) \sin \left(a_{1} e_{1}\right),
\end{array}\right\}
$$

where

$$
\begin{aligned}
& D\left(e_{1}\right)=e_{i}^{2} \sum_{i=2}^{10} \frac{D_{i}}{M_{i}} \\
& P\left(e_{1}\right)=e_{1} \sum_{i=2}^{10}\left(\frac{P_{\mathrm{m} i}}{M_{i}}-\frac{G_{i i} E_{i}^{2}}{M_{i}}-\sum_{j=2, j \neq i}^{10} \frac{E_{i} E_{j} G_{i j}}{M_{i}}\right) \\
& G\left(e_{1}\right)=e_{1} \sum_{i=2}^{10} \frac{E_{i} E_{1} G_{i 1}}{M_{i}} \\
& B\left(e_{1}\right)=e_{1} \sum_{i=2}^{10} \frac{E_{i} E_{1} B_{i 1}}{M_{i}} .
\end{aligned}
$$

The above system (9) corresponds to the well-known dynamical system that describes swing dynamics of single generator connecting to the infinite bus via a lossy transmission line [8]. The system has the potential function $U\left(a_{1} ; e_{1}\right)$, given by

$$
\begin{aligned}
U\left(a_{1} ; e_{1}\right) & =-P\left(e_{1}\right) a_{1} \\
+ & \frac{G\left(e_{1}\right)}{e_{1}} \sin \left(a_{1} e_{1}\right)-\frac{B\left(e_{1}\right)}{e_{1}} \cos \left(a_{1} e_{1}\right) .
\end{aligned}
$$

The system (9) reveals a dynamical mechanism responsible for the occurrence of global instability in Fig. 2. Fig. 4(b) clearly shows that the coefficient $a_{1}(t)$ diverges after a finite number of bounded swings. The divergence of $a_{1}(t)$ affects each rotor angle position $\delta_{i}(t)$ in a uniform manner, because the base of the first POD mode is almost flat. Here we recall that the dynamical mechanism behind divergence motions of the system (9) is the escape from a potential well for highenergy regime, which is extensively studied in [6]. Therefore we can conclude that the dynamical mechanism responsible for the instability is the escape from a potential well in the dynamical system for the time-varying coefficients of the first POD mode. This is the same mechanism as that in the preceding paper [1]. Thus the dynamical mechanism of instability can be determined with the help of POD and the Galerkin method.

\section{SUMMARY AND DISCUSSION}

This paper analyzed global instability in the New England grid model. By the POD and the Galerkin method, we show that the global instability in Fig. 2 is the escape phenomenon in the dynamical system for the first POD mode and, that is, has the same mechanism as that proposed in [1]. Applying these methods to power grid analysis is not a new approach and is reported in [12], [13]. The contribution of this paper is to show that these methods are capable of determining a dynamical mechanism responsible for global instability of the general classical model (1).

It is meaningful to discuss whether the application of the POD and the Galerkin method is generalized. The POD itself is a general method and is applicable to data obtained with not only numerical simulation of general dynamic models, including the detailed model in Sec. II-B, but also measurement in a real power grid. The Galerkin method is also used in [12] for a high-dimensional dynamic model and is applicable to a dynamic model with topology changes. These methods thus have a great potential for exploring global instability in complex power grids. This enables us to find the occurrence of global instability using data measured in a real grid.

A limitation of the application should be also discussed. The Galerkin method uses simulation outputs of swing dynamics. A model derived by the method hence depends strongly on the simulation and mathematical formula of the dynamic model used there. This implies that the accuracy of the reduced model depends on the data and mathematical models. This may give a limitation of the application. It is significant to choose proper outputs and mathematical models in order to achieve successful reduction in the sense that the obtained low-dimensional model approximates the phenomenon accurately.

The obtained result is also related to coherency analysis in stability estimation using energy functions method [8], [14]. Energy functions method is based on potential structures of dynamic models for power grid stability analysis. The POD can identify a group of generators in which they behave in a coherent manner. The Galerkin method makes it possible to find a hidden potential structure for the group of generators. These methods can hence contribute to development of energy functions method that can take the group of generators into account.

\section{ACKNOWLEDGMENTS}

The first author is grateful to Professor Yasuharu Ohsawa (Kyoto University) and Professor Tsuyoshi Funaki (Osaka University) for valuable instructions of power grid computation. The authors are also grateful to anonymous reviewers for their careful reading of the manuscript. 


\section{REFERENCES}

[1] Y. Susuki, I. Mezić, and T. Hikihara, "Global swing instability of multimachine power systems," in Proceedings of the 47th IEEE Conference on Decision and Control, Cancun, Mexico, December 911 2008, pp. 2487-2492.

[2] P. Kundur, Power System Stability and Control. McGraw-Hill, 1994.

[3] G. Andersson, P. Donalek, R. Farmer, N. Hatziargyriou, I. Kamwa, P. Kundur, N. Martins, J. Paserba, P. Pourbeik, J. Sanchez-Gasca, R. Schulz, A. Stankovic, C. Taylor, and V. Vittal, "Causes of the 2003 major grid blackouts in North America and Europe, and recommended means to improve system dynamic performance," IEEE Transactions on Power Systems, vol. 20, no. 4, pp. 1922-1928, November 2005.

[4] B. Avramovic, P. V. Kokotovic, J. R. Winkelman, and J. H. Chow, "Area decomposition for electromechanical models of power systems," Automatica, vol. 16, pp. 637-648, 1980.

[5] S. Varigonda, T. Kalmár-Nágy, B. LaBarre, and I. Mezić, "Graph decomposition methods for uncertainty propagation in complex, nonlinear interconnected dynamical systems," in Proceedings of the 43rd IEEE Conference on Decision and Control, Pradise Island, Bahama, December 14-17 2004, pp. 1794-1798.

[6] J. M. T. Thompson, "Chaotic phenomena triggering the escape from a potential well," Proceedings of the Royal Society of London. A, Mathematical and Physical Sciences, vol. 421, pp. 195-225, 1989.

[7] T. Athay, R. Podmore, and S. Virmani, "A practical method for the direct analysis of transient stability," IEEE Transactions on Power Appratatus and Systems, vol. PAS-98, no. 2, pp. 573-584, March/April 1979.

[8] M. A. Pai, Energy Function Analysis for Power System Stability. Kluwer Academic Pub., 1989.

[9] P. Holmes, J. L. Lumley, and G. Berkooz, Turbulence, Coherent Structures, Dynamical Systems, and Symmetry. Cambridge: Cambridge University Press, 1996.

[10] Y. Ohsawa, "Studies on applications of Lypunov's direct method to power system stability analysis," PhD Dissertation, Kyoto University, December 1981, (in Japanese).

[11] IEEE Recommended Practice for Excitation System Models for Power System Stability Studies, IEEE Std. 421.5-1992, 1992.

[12] P. A. Parrilo, S. Lall, F. Paganini, G. C. Verghese, B. C. Lesieutre, and J. E. Marsden, "Model reduction for analysis of cascading failures in power systems," in Proceedings of the American Control Conference, San Diego, June 1999, pp. 4208-4212.

[13] K. K. Anaparthi, B. Chaudhuri, N. F. Thornhill, and B. C. Pal, "Coherency identification in power systems through principle component analysis," IEEE Transactions on Power Systems, vol. 20, no. 3, pp. 1658-1660, August 2005.

[14] H. D. Chiang, C. C. Chu, and G. Cauley, "Direct stability analysis of electric power systems using energy functions: Theory, applications, and perspective," Proceedings of the IEEE, vol. 83, no. 11, pp. 14971529, November 1995. 